\title{
Laser Patterning of Thin Film Copper and ITO on Flexible Substrates for Terahertz Antenna Applications
}

\author{
Manikandan Esakkimuthu*, Sreeja Balakrishnapillai Suseela*, Radha Sankararajan*, Abhishek Gupta**, Goutam Rana** \\ and Prabhu S. ${ }^{* *}$ \\ ${ }^{*}$ Department of Electronics and Communication Engineering, SSN College of Engineering \\ Chennai, India-603110 \\ E-mail: manikandane@ssn.edu.in \\ ${ }^{* *}$ Tata Institute of Fundamental Research,Homi Bhabha Road \\ Mumbai, India-400005 \\ *** Indian Institute of Technology \\ Mumbai, India-400076
}

\begin{abstract}
This work demonstrates the functioning of creating a micro-scale patch structure in copper and optically transparent Indium Tin Oxide thin film by a mode-locked 20ns and 650ps laser pulses with 1064nm and 532nm wavelength respectively. The proposed device uses $\mathrm{Cu}$ and ITO as conducting patches. A thin film of 0.4 micron $\mathrm{Cu}$ and 0.125 micron ITO was deposited on Polyimide and PET substrate respectively. The crystalline of the deposited $\mathrm{Cu}$ and ITO films have been discussed. It is clear that the ablation threshold is dependent on applied power, pulse width, and scanning speed. The analysis of the results has been done using Scanning Electron Microscope, an Optical Profiler, and a white line interferometer. The ablation depth of the patch profiles was analyzed through an optical profiler. The temperature rise in the ITO by the applied laser pulse has been studied using FEM simulation and the laser ablation of ITO film from PET substrate by the delamination process was observed. The created structure finds application in Terahertz Antenna in making infrared detector and the performance metrics of the developed structures are studied using EM simulation software. The resonance nature of the fabricated structure has been validated experimentally by THz-TDS technique.

DOI: 10.2961/jlmn.2017.03.0023
\end{abstract}

Keywords: copper, ito, laser, micromachining, transparent, antenna, terahertz

\section{Introduction}

Advancements in wireless technologies many challenges have been seen in the designing highspeed communication devices such as Resonators, Filters, and Antennas etc.Though Microwave and Millimeter technology have been in research, the entire spectrum becomes congested by more utilization. The $\mathrm{THz}$ radiation i.e. far infrared range is non-ionizing and more room is available for utilization.FIR devices are mainly used in security, imaging and IR harvesting applications [1].

Integration of micro strip antennas with solar cells with a minimal area for space applications has attracted research attention. But Integration of antennas with solar cell has a lot of challenges such as optical transparency, antenna performance, compatibility etc. Meshed patch antennas are reported in the literature for providing optical transparency [2]. Alternatively, antennas fabricated on a polymer substrate with transparent conducting oxide (ITO) patch facilitate integration with solar cell without any transparency issues [3]. At the same time, ITO-based patch antenna exhibits a high bandwidth operation also [4].

If the antenna is able to operate at infrared frequencies and with a suitable diode for AC to DC conversion, it can be used for enhancing the performance of the solar cell by extending their absorption band to infrared, thereby improving the efficiency of the cell [5].

At these frequencies, the size of the devices will be in a micron scale and hence integrating these devices (Filter, Antennas etc) with solar cell does not have any effect on the performance of the cell with no deterioration in its own performance. But the major problem lies in the fabrication of micro and nano scaled devices. Generally, it requires high-end equipments like E-beam lithography, RIE techniques and also a clean room environment.

A Microstrip patch consists of three layers, namely, ground plane, a dielectric substrate and a conducting patch made up of copper. Based on the created patterns on the patch and the size it resonates at particular frequency [6]. In this work nanosecond and picoseconds, laser sources have been utilized for creating the required patch structure for antenna application. Currently, Laser Micromachining is used in many applications for fabrication of micro/nano structures. Here the objective is to ablate and remove the materials from the required area by laser micromachining.

Nanosecond laser ablation of metals creates a highly heat affected zone and more molten regions compared to short pulse lasers such as picoseconds and femtosecond lasers. Laser material processing photochemical ablation process occurs in 
picoseconds since the thermalization time is comparatively larger than the excitation time of the laser source [7].

In literature, both theoretical modeling and experimental investigations of copper, nickel, silver targets have been studied for various laser sources UV laser, Nd: YAG lasers (nanoseconds, picoseconds and femtosecond) for laser beam characteristics (Fluence, wavelength, pulse duration) at different environment conditions [8-13].Recently, the use of AOD's (acoustic-optic deflectors) in laser beam steering approach for the micro-structuring copper layer has been reported [14]. It is also possible to develop flexible electrode for display applications by nanosecond laser ablation.

Nomenclature

k-Thermal conductivity(W/m- K)

C-specific heat $(\mathrm{J} / \mathrm{kg}-\mathrm{K})$

$\rho$-Mass density $\left(\mathrm{kg} / \mathrm{m}^{3}\right)$

$\alpha$-Absorption coefficient $\left(\mathrm{m}^{-1}\right)$

l- Optical absorption length(nm)

R-Reflectivity used in the model

$\mathrm{l}_{\mathrm{t}}$-Thermal Diffusion Length (nm)

$T_{m}$-Melting point (K)

$T_{v}(\mathrm{~K})$-Vaporization Temperature

$\mathrm{L}_{\mathrm{m}}$-Latent Heat of fusion, $(\mathrm{J} / \mathrm{Kg})$

$\mathrm{L}_{\mathrm{v}}$-Heat of vaporization $(\mathrm{J} / \mathrm{Kg})$

$\mathrm{IP}_{1}$-First ionization potential, $(\mathrm{eV})$

$\varphi(\mathrm{eV})$-Electronic work function

E -Young's Modulus (GPa)

V- Poisson's Ratio

CTE- Coefficient of Thermal Expansion $\left(\mathrm{K}^{-1}\right)$

In recent years, ITO thin film finds many applications, display devices, an electrode for the solar cell, touch panels etc. So selective removal of ITO layer from the substrate has gained considerable attention. Several theoretical, numerical and experimental works have been done on ITO thin film removal, coating and reported in the literature for various pulses and laser beam characteristics [1519].In this work selectively etched ITO is used as a patch layer in the antenna design. C. McDonnell et al. have examined de-lamination as potential film removal mechanism for ITO thin films using ns pulsed laser [20]. In nanosecond laser pulse, removal induces more thermal damage to the film. The influence of laser pulse duration, scanning speed and laser Fluence for ITO film of few microns for narrow grooves using femtosecond laser has been investigated [21]. The removal of thin film ITO using nanosecond and femtosecond laser pulse has been studied at different wavelengths and pulse duration. The deposition of ITO thin films on polymer substrate was accomplished by magnetron sputtering and its optical properties have been analyzed using UV-VIS spectroscopy.
The major objectives of this work are to investigate the role of nanosecond and picoseconds lasers Fluence and scanning speed and laser Fluence at wavelengths of $1064 \mathrm{~nm}$ and $532 \mathrm{~nm}$ on selective removal of copper and ITO from the substrate.

The developed heat during laser ablation along with stress distributions in the thin film has also been studied using FEM simulations tool. In this paper, the 20ns and 650ps laser sources Fluence and scanning speed on ablation rate of copper and ITO are described. The peak temperature and its distribution through the surface are obtained using FEM simulation. Finally, the simulated performance of the ablated structure for the antenna is studied and discussed.

\section{Experiments Details}

A patch antenna has three layers; a ground plane (Copper), a dielectric substrate (PET, Polyimide) and a patch (Indium Tin Oxide, Copper).ITO is a transparent conducting oxide and chosen as a conducting patch material in this work. While integrating with solar cell applications the ground plane could be replaced with the solar cell back electrode for providing a fully transparent antenna. The pattern to be generated on copper and ITO by laser ablation process is shown in Fig. 1 (white area to be etched away by the laser pulses).
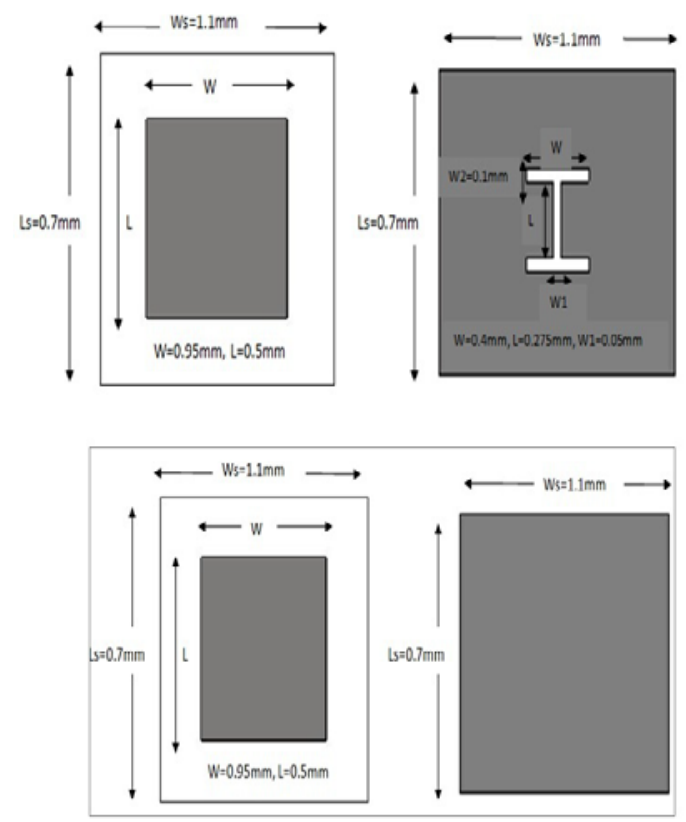

Fig.1 Pattern to be ablated by laser a) Copper Patch b) ITO Patch

\subsection{Materials}

A thin film copper was deposited on $0.125 \mathrm{~mm}$ thick polyimide material and the measured thickness of the deposited copper was $0.4 \mu \mathrm{m}$.Since the pattern targeting $\mathrm{THz}$ antenna applications, the thickness of the deposited copper should be greater than the skin depth value. The skin depth value at $1 \mathrm{THz}$ was $0.0652 \mu \mathrm{m}$ which was comparatively smaller than the 
thickness of the copper layer deposited on the polyimide substrate $(0.4 \mu \mathrm{m})$. So the fabricated antenna is not susceptible to skin effect.

An XRD analysis was taken for the deposited copper film. The resultant graph shows that the copper has <111> crystalline structure at $2 \theta=42.8^{\circ}$.Polyimide peaks are also seen in the result. Again the deposition of ITO thin films on PET substrate was accomplished and its crystalline characteristics, optical properties were analyzed by XRD and UV-VIS spectrometer. X-Ray Diffractions shows (Fig.2) that the ITO has a broad pattern at $2 \theta=30^{\circ}$ with amorphous in nature [18]. The properties of the materials used are listed in the Table 1.

Table1 Properties of the Materials used in this work

\begin{tabular}{|c|c|c|}
\hline $\begin{array}{l}\text { Parame } \\
\text { ters } \\
\rho \\
\end{array}$ & $\begin{array}{c}\text { ITO- } 340 \text { IT } \\
1064 \mathbf{n m} \\
71 \text { OSO32nm } \\
\end{array}$ & $\begin{array}{l}\text { 420(ropliper)(1064 } \\
\text { moy (liquid) } \\
\text { 8960(solid), }\end{array}$ \\
\hline $\mathrm{k}$ & 10 & 88800(sdiligidij,d) \\
\hline $\mathrm{a}$ & $10^{6}$ & $17 \sigma(4 i t)<10)$ \\
\hline l & $5^{126}$ & -- \\
\hline $\mathrm{R}$ & $0.01^{(0.2)}$ & 0.34 \\
\hline $\mathrm{l}_{\mathrm{t}}$ & 275 & -- \\
\hline$T_{m}$ & 1900 & 1357 \\
\hline$T_{v}$ & 3000 & 2835 \\
\hline $\mathrm{L}_{\mathrm{m}}$ & $5 \times 10^{5}$ & $1.3 \times 10^{4}$ \\
\hline $\mathrm{L}_{\mathrm{v}}$ & $5 \times 10^{6}$ & $3.048 \times 10^{5}$ \\
\hline $\mathrm{IP}_{1}$ & 4.76 & 7.73 \\
\hline$\varphi$ & 3.65 & 4.5 \\
\hline E & 190 & 128 \\
\hline V & 0.35 & 0.34 \\
\hline CTE & $5 \times 10^{-6}$ & $16.5 \times 10^{-6}$ \\
\hline
\end{tabular}

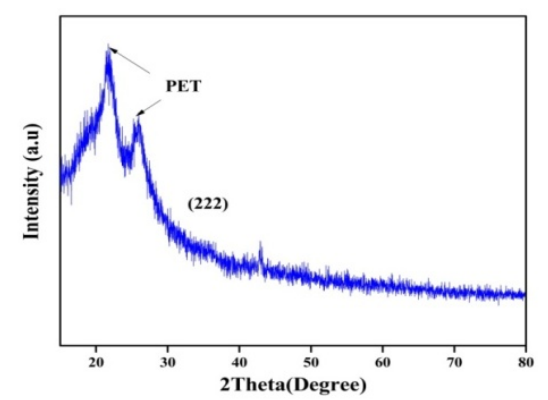

Fig.2 X-ray diffraction pattern of ITO thin film over PET substrate

The absorption coefficient of a thin film ITO can be determined by using Beer-Lambert law(1),

$\alpha(\lambda)=\left(-\frac{1}{t} \ln \left[T(\lambda) /\left\{\left(1-R^{\prime}(\lambda)\right)(1-R(\lambda))\right\}\right]\right)(1)$
Where $\mathrm{t}$ is the film thickness, $T(\lambda)$ is the measured transmission, $R(\lambda)$ measured reflectivity of air/ITO interface and $R^{\prime}(\lambda)$ is the estimated reflectivity of PET/ITO interface. The refractive index of constant value 1.46 is taken for PET substrate. The estimated reflectivity of the ITO/PET interface was determined by Fresnel equation,

$$
R^{\prime}(\lambda)=\left(\left(\eta_{\text {ITO }}-\eta_{p}\right)\left(\eta_{\text {ITO }}+\eta_{p}\right)\right)^{2}
$$

This resulted in the estimated reflectivity at $1064 \mathrm{~nm}$ wavelength gives 0.01 and the corresponding absorption coefficient as $1.1 \times 10^{6}\left(\mathrm{~m}^{-1}\right)$ [16].The input parameters of the laser sources used for ablation are listed in Table 2.These parameters are utilized for ns-pulsed ablation of ITO and copper using FEM simulation.

Table 2 Laser Input Parameters

\begin{tabular}{ccccc}
$\begin{array}{l}\text { Waveleng } \\
\text { th } \\
\text { (nm) }\end{array}$ & $\begin{array}{l}\text { Pulse } \\
\text { durati } \\
\text { on }\end{array}$ & $\begin{array}{l}\text { Repetiti } \\
\text { on rate } \\
\text { (KHz) }\end{array}$ & $\begin{array}{l}\text { Raw } \\
\text { Beam } \\
\text { Output(m } \\
\text { m) }\end{array}$ & $\begin{array}{l}\text { Focuss } \\
\text { ed Spot } \\
\text { radius } \\
\text { ( mm) }\end{array}$ \\
\hline 1064 & $20 \mathrm{~ns}$ & 30 & 0.9 & 0. \\
\multicolumn{1}{c}{532} & $650 \mathrm{ps}$ & 45 & 2.0 & \multicolumn{2}{c}{0.} \\
\hline
\end{tabular}

\subsection{Pattern Generation and Analysis}

The experiments were carried out at different scanning speeds and power level for nanosecond pulsed laser (SPI Lasers) and at single scanning speed with three power levels for picoseconds laser pulses (PicoSpark ${ }^{\mathrm{TM}}$ ). A constant wavelength of $1064 \mathrm{~nm}$ and 532nm was utilized for ns and ps sources. The block diagram of the used picoseconds laser setup is shown in the figure.

The setup consisted of a ps pulsed laser, turning mirrors, attenuator, expander, galvanometer and a focusing lens. The simplified block diagram of the picoseconds laser used in the experiments is shown in Fig.3.

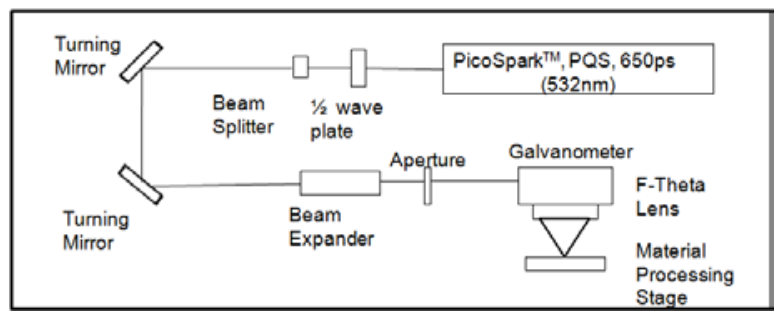

Fig.3 650ps-pulsed Laser setup used in the experiment

\subsubsection{ITO Patch}

Findings from literature show a minimum amount of laser energy supplied to ablate ITO film from the PET substrate. The observations trigger variations in laser Fluence for nanosecond pulse from $(0.22-1.3) \mathrm{J} / \mathrm{cm}^{2}$ at a constant scanning speed of $1000 \mathrm{~mm} / \mathrm{sec}$ is applied to the ITO film on PET substrate and the corresponding ablation depth values have been plotted. The ablation depth for different 
fluencies was obtained using an optical surface profiler(BRUKER, ConourGTInMotion).

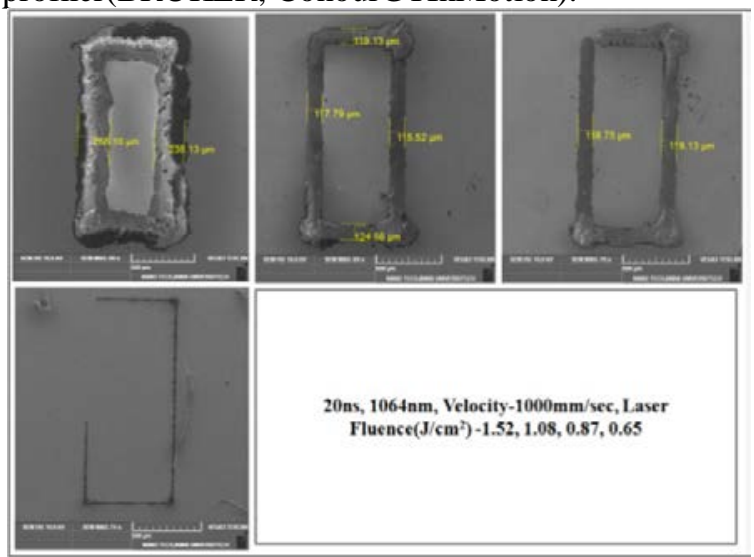

Fig.4 SEM Images of the ablated samples at different Fluence by 20ns 1064nm pulsed laser

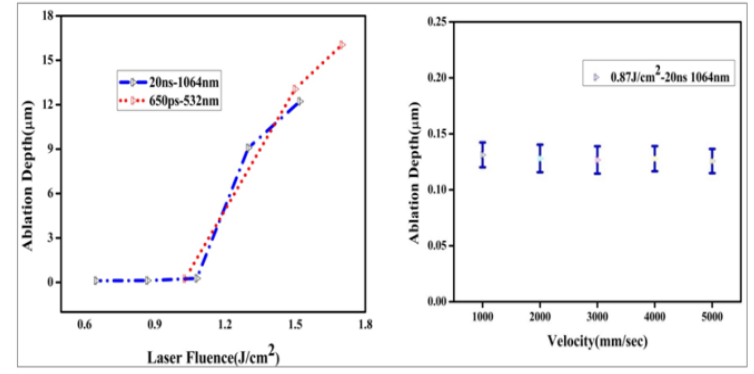

Fig.5 Laser Fluence vs. Ablation depth for ns and ps pulse and velocity vs. ablation depth for 20ns pulse at constant Fluence

The SEM images of the ablated samples (ITO) attained using a 20ns pulsed laser at different Fluence levels are shown in Fig.4.At a higher Fluence the heat affected became high compared to the lower Fluence. But at high energy density the melted samples extended the ablation by thermal diffusion process and the ablation volume increased.Fig.4 depicts the plot between applied laser Fluence $\left(\mathrm{J} / \mathrm{cm}^{2}\right)$ and ablation depth $(\mu \mathrm{m})$. The graph shows that a constant value of $(0.65-0.87) \mathrm{J} / \mathrm{cm}^{2}$ is sufficient to remove the required thin film ITO from the PET substrate. But at this Fluence level, with $1000 \mathrm{~mm} / \mathrm{sec}$ scanning speed the heat affected zone was seen as much higher than expected and edges were not uniform in the entire area. That was, therefore the necessity to optimize the scanning speed of the laser to improve the ablation with fine quality edges. A range of scanning speed from $1000 \mathrm{~mm} / \mathrm{sec}$ to $5000 \mathrm{~mm} / \mathrm{sec}$ was taken for analysis. For these ranges with constant energy, there was not much difference seen in the ablation volume but uniformity was not the same. The figure shows the plot of applied Fluence vs. ablation depth for ns and ps pulsed laser. Fig. 5 also includes the error values(y-axis) for ns pulsed laser at $0.87 \mathrm{~J} / \mathrm{cm}^{2}$ for different scanning velocity at three iterations.

In picoseconds laser process, initially; a small dot and line were tried before starting the pattern generation. Different scanning speeds were tried at a constant power level. The figure shows the corresponding optical microscopic image for a line and dot created with 650ps laser pulse at constant power. The heat affected zone due to thermal diffusion was much higher than the spot diameter of the laser pulse, showing formation of wrinkles around it. Then an optimum scanning speed of $500 \mathrm{~mm} / \mathrm{sec}$ was chosen for the ablation process. The corresponding optical microscopic view and WLI images of the dot and line pattern of the ablated samples are shown in Fig.6.

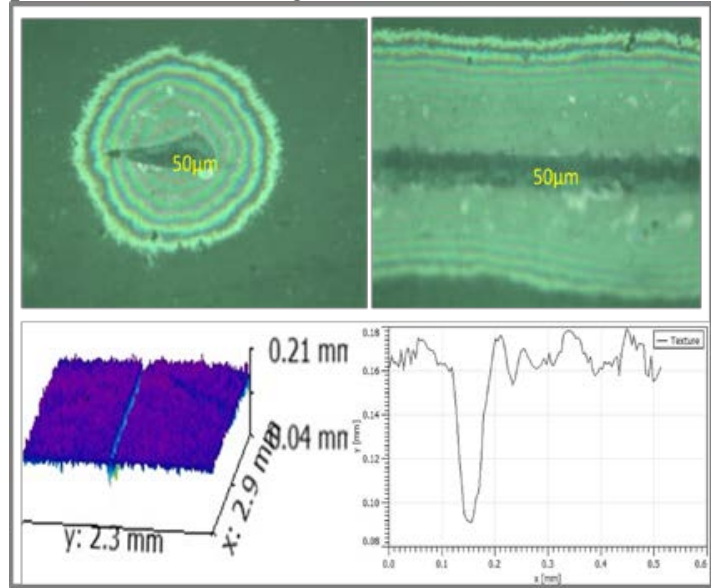

Fig.6 Optical microscopic view of the dot, line created and WLI image of the corresponding line pattern

Following the initial analysis, optical surface profiler was used for measuring the ablation depth at a constant scanning speed of $500 \mathrm{~mm} / \mathrm{sec}$ for three Fluence levels and the surface morphologies were studied using SEM and WLI.Fig.7 shows the WLI image of the patch created with the ps-pulsed laser at the velocity of $500 \mathrm{~mm} / \mathrm{sec}$ at $0.52 \mathrm{~J} / \mathrm{cm}^{2}$.
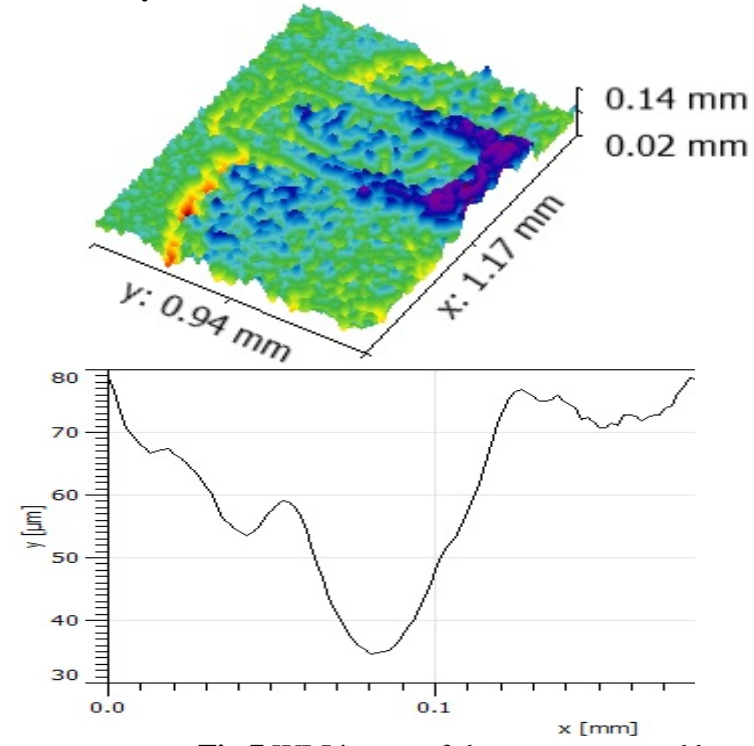

Fig.7 WLI image of the pattern created by ps-pulsed laser and corresponding 2D ripple in the cut area

\subsubsection{ITO Temperature Simulation}

The information relating to peak temperature and temperature distributions were obtained numerically using the finite element simulation. The observed 
peak temperature for 20ns 1064nm wavelength was about $1200 \mathrm{~K}$. A uniform distribution of heat through the surface as a result of thermal diffusion observed. The properties of the material used in the simulation are listed in Table 1. But after 10ns of the time period, the temperature became high enough to melt and vaporize the material. A stress due to the thermal process was generated at the PET substrate for the applied laser pulses and delaminating occurs at the surface. This led to the removal of ITO film from the substrate. The temperature distribution at 20ns for the ITO patch is shown in Fig.8.

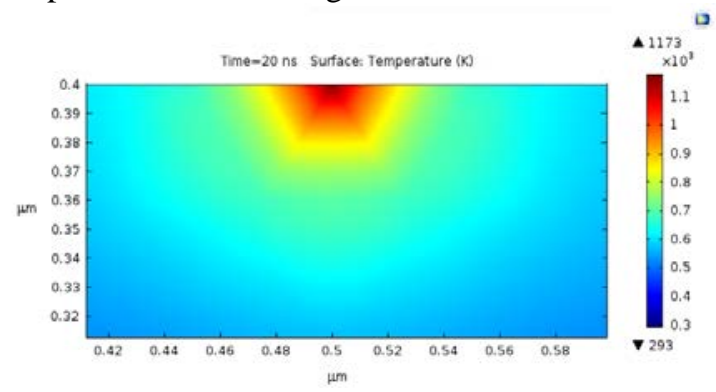

Fig.8 Temperature Distribution through ITO film for ns-pulsed laser ablation.

The transversal and vertical thermal diffusion length for ITO for $20 \mathrm{~ns}$ pulse is about $0.2 \mu \mathrm{m}$ which is best matches with the properties listed in Table 1 .

\subsubsection{Copper Patch}

Analysis of the surface morphology of the ablated patterns was done using by Scanning Electron Microscope (TESCAN VEGA3) while the ablation depth was measured using an optical profiler (BRUKER, ConourGT InMotion) and White Line Interferometer. The WLI images of the ablated patterns and their corresponding 2D ripple profile of the cut cross sections are shown in Fig.9. Initially WLI images were taken for the samples treated at $10.83 \mathrm{~J} / \mathrm{cm}^{2}$ for confirming the pattern generation. For the I-shaped pattern, 2d ripple profile was taken at the mid of the I portion. The nanosecond laser sources did removal by the photothermal process so a nominal heat affected zone near the ablation area was observed from the figure.

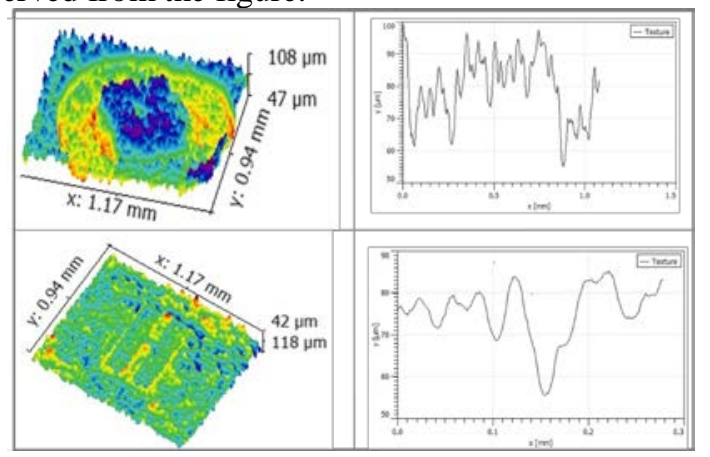

Fig.9 WLI images of the sample treated by 20 ns laser pulse at $10.83 \mathrm{~J} / \mathrm{cm}^{2}$ Fluence a) Square pattern around a patch b) I shape.

In picoseconds laser ablation, a line was marked on the copper sample with the same laser for the Fluence level at $13.79 \mathrm{~J} / \mathrm{cm}^{2}$ at two different scanning speed of $100 \mathrm{~mm} / \mathrm{sec}$ and $500 \mathrm{~mm} / \mathrm{sec}$ before the creation of the required pattern. The optical microscopic view of the marked samples and their corresponding WLI images are shown in Fig.10. A smooth line with minimal heat affected area was obtained at a velocity of $500 \mathrm{~mm} / \mathrm{sec}$. The laser pulse had the ability to ablate the line width of $50 \mu \mathrm{m}$ with the depth of around 100 microns. The entire surface of the device was seen ablated for the Fluence used. Here the observation was that the laser Fluence should be maintained below this levels and nominal values require consideration for the pattern generation.

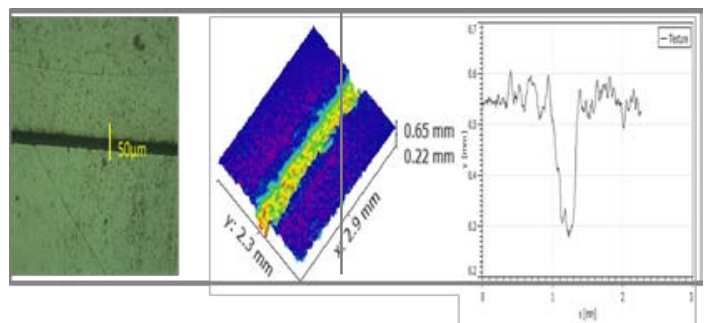

Fig.10 Optical Microscopic view of the initial line created by picoseconds Laser at $100 \mathrm{~mm} / \mathrm{min}$ and WLI images for the sample.

Using the findings from the above analysis and discussions, the laser Fluence was calculated for nanosecond laser pulse and a range of $(2-13) \mathrm{J} / \mathrm{cm}^{2}$ was chosen for the generation of the required pattern at six different scanning speeds. Similarly, for picoseconds pulse three different laser Fluence (3-6), $\mathrm{J} / \mathrm{cm}^{2}$ at a constant scanning speed of $100 \mathrm{~mm} / \mathrm{sec}$ have been utilized in this work.

Fig.11 shows the SEM images of the patterned samples at $1000 \mathrm{~mm} / \mathrm{sec}$ for different Fluence levels for ns pulsed source. At the maximum Fluence of $10.83 \mathrm{~J} / \mathrm{cm}^{2}$ a random ablation occured on the surface i.e. more area was ablated without any uniformity is obtained. In another case with the lowest Fluence of $2.17 \mathrm{~J} / \mathrm{cm}^{2}$, clean structures were formed with smaller heat affected area

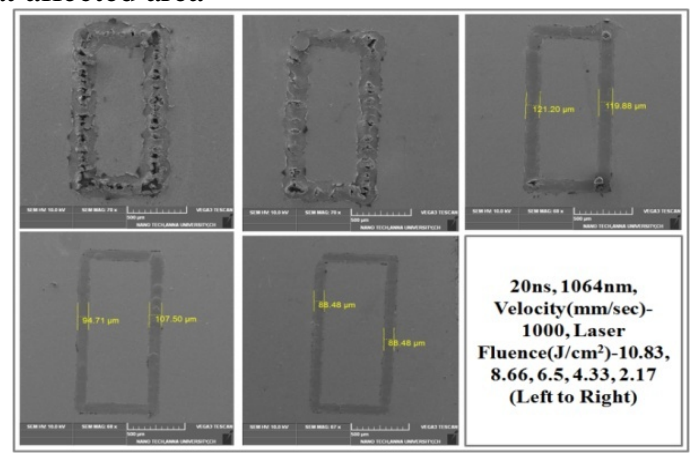

Fig.11 SEM Images of the Patch created with 20ns-pulsed laser 

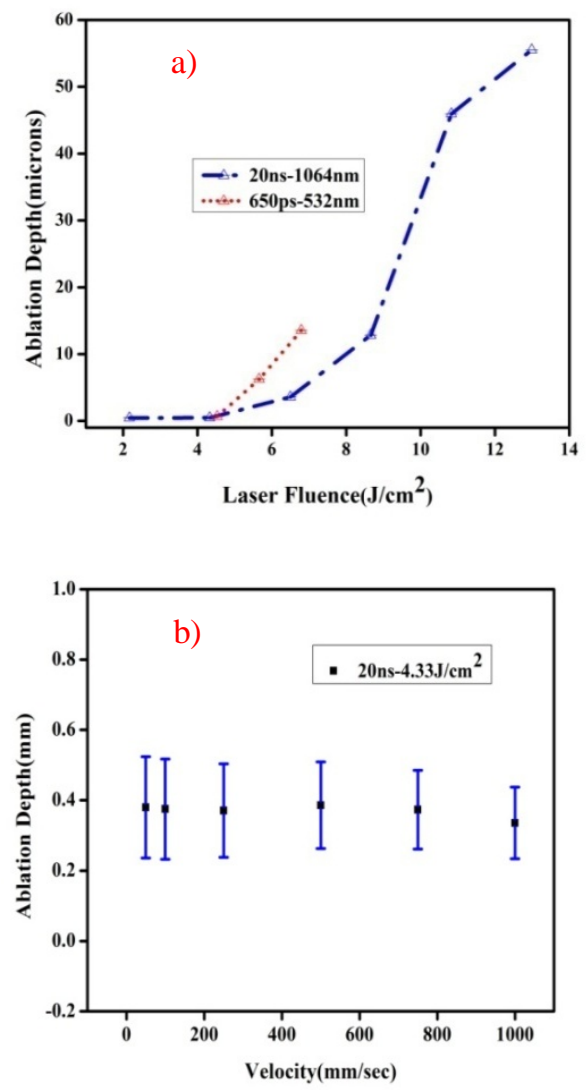

Fig. 12 a) Laser Fluence vs. Ablation depth (ns and ps laser) b) velocity vs. ablation depth for 20ns pulsed laser at constant Fluence.

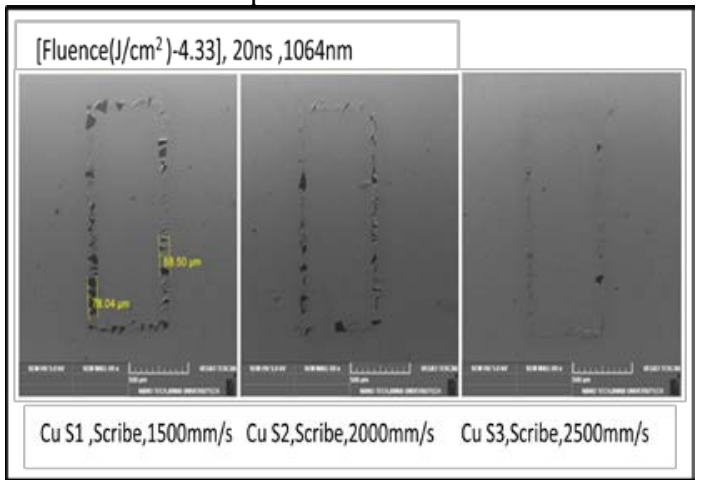

Fig.13 SEM Images of the ns laser ablated copper at different scanning speed

The laser Fluence vs. ablation depth at a constant scanning speed of $1000 \mathrm{~mm} / \mathrm{sec}$ and scanning velocity vs. ablation depth at a constant Fluence are plotted and shown in the graph Fig.12 a).A comparatively small energy was required for picoseconds laser for ablation of the same depth, this could be due to a small focussing spot and the entire laser irradiation was converged at the required area in the ps-pulsed source. Another important point is that, in material, especially in metals, the energy relaxation time is in the order of $10^{-13} \mathrm{~s}$. So the optical energy is converted and treated as a heat source in nanosecond source but with ps-pulsed, this is not the case. Fig.12.b) shows the velocity vs. ablation depth at a constant Fluence of $4.33 \mathrm{~J} / \mathrm{cm}^{2}$ for ns pulsed laser for different trials and the error values are included in the plot. After $1000 \mathrm{~mm} / \mathrm{sec}$ the patterns are not uniformly etched from the substrate. Fig.13. shows the corresponding SEM images at $1500 \mathrm{~mm} / \mathrm{sec}, 2000 \mathrm{~mm} / \mathrm{sec}$ and $2500 \mathrm{~mm} / \mathrm{sec}$. No ablation was seen when scanning speed was increased and smooth ablation was not expected.So a smooth pattern with minimal heat affected area was obtained at optimized velocity $(1000 \mathrm{~mm} / \mathrm{sec})$ and Fluence $\left(4.33 \mathrm{~J} / \mathrm{cm}^{2}\right)$,.

\subsubsection{Copper Temperature Simulation}

Information relating to the peak temperature and its distribution through the film was extracted using the finite element simulation. A 20ns 1064nm laser source was modeled for this purpose and given as an excitation to the copper thin film of thickness $0.4 \mu \mathrm{m}$. The peak temperature observed at 20ns was approximately 2300K.Since the temperature exceeded the melting point of the copper molten target started appearing on the surface. But between $20 \mathrm{~ns}$ to $30 \mathrm{~ns}$ the temperature started increasing further and reached about $4750 \mathrm{~K}$ which was high enough to vaporize the material. Here only the temperature distribution information's were obtained and the phase changes are not included in the simulation. This temperature simulation is matched reasonably with the mathematical approach reported earlier in the literature [14]. The contour temperature distribution at 20ns is shown in Fig.14.

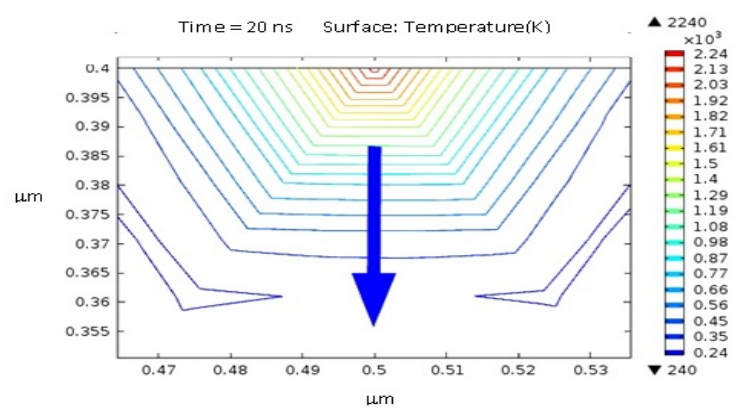

Fig.14 Temperature Distribution through copper and ITO thin film for 20ns pulsed laser

\section{THz Antenna Design and Numerical Evaluation}

The antenna consists of a patch layer printed on a dielectric substrate with a perfect ground plane. At higher frequencies spurious resonant made its appearance as a result of the impedance mismatch problem and it affected the performance of the antenna (By theory, the patch antenna has dual-band operation).In this case, a spurious resonance appeared at $0.23 \mathrm{THz}$ which was not a harmonics also. So a defect was introduced in the ground plane -DGS (Defective Ground Structure) which could suppress the spurious signal. As shown Fig.1 with the introduction of DGS, the resonant showed improvement at the desired frequency $0.15 \mathrm{THz}$ and its performance characteristics were studied and 
discussed. For the ablated pattern, from theory, the resonant frequency of the antenna had its dependence on the width and length of the patch size.From operating frequency, the width and the length of the patch were given by (1) and (2) respectively. It is understood that the fabricated structure will be resonating at $0.15 \mathrm{THz}$.

$$
L=\frac{C}{2 f_{r} \sqrt{\varepsilon_{r}}}
$$

The directivity and gain of the DGS antenna improved at $0.15 \mathrm{THz}$, the radiation characteristics of the designed antenna are shown in Table 3. Thus the patterned structure behaves as a $\mathrm{THz}$ antenna and can be used in imaging, wireless applications for improving the sensitivity and data rate. The return loss characteristics of the patterned antenna are shown in Fig.15.There was no effect on the resonant frequency with the DGS pattern.

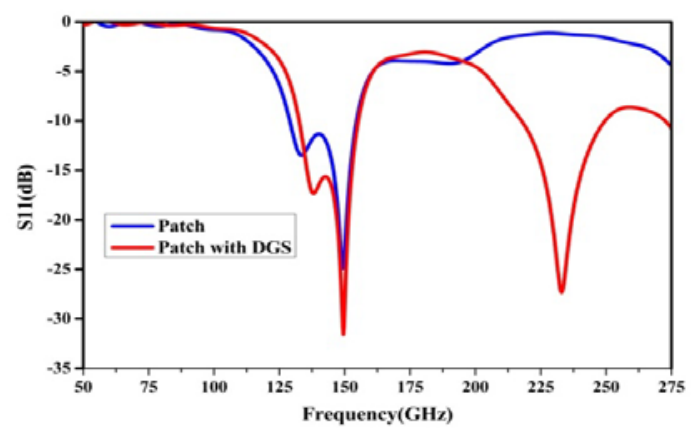

Fig.15 S11(dB)-Return Loss characteristic of the patterned antenna

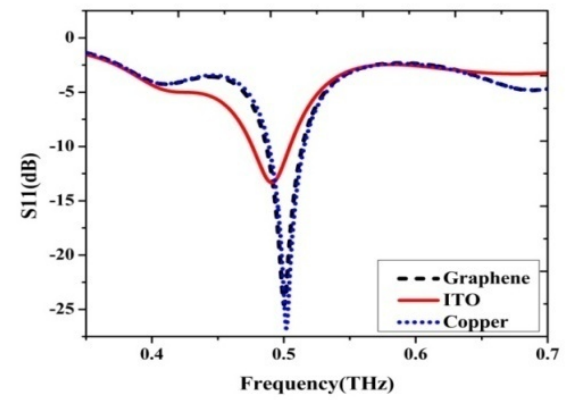

Fig.16 Return Loss characteristics of the designed antennas

Table 3 Radiation Characteristics of the Laser patterned antenna

\begin{tabular}{c|l|l|c|l}
\hline Type & $\begin{array}{l}\text { Frequency } \\
\text { (THz) }\end{array}$ & $\begin{array}{l}\text { S11 } \\
\text { (dB) }\end{array}$ & $\begin{array}{l}\text { Gain } \\
\text { (dB) }\end{array}$ & $\begin{array}{l}\text { D } \\
(\mathbf{d B})\end{array}$ \\
\hline Patch & $0.15,0.233$ & $\begin{array}{l}-31.46, \\
-27.22\end{array}$ & 5.17 & 5.3 \\
Patch - & 0.15 & $\begin{array}{l}-23.37 \\
\text { DGS }\end{array}$ & 6.23 & 6.42 \\
\hline
\end{tabular}

The objective of this fabricated patch structure was its use as an antenna operating at the $\mathrm{THz}$ frequency. So, simulation was done for this structure using Electromagnetic software. The simulated return loss (S11) is shown in the graph (Fig.16). The generated pattern resonates at $0.5 \mathrm{THz}$ and provide a return of loss -25dB.The value obtained for ITObased structure $(-15 \mathrm{~dB})$ is seen as less than copper based conventional antenna but it provides optical transparency to enable integration with a solar cell for space application to reduce the size of the device. The obtained radiation characteristics are shown in Table 4.The dependence of operating frequency on the size of the patch and the properties of the dielectric substrate was theoretically verified. The following equations justify the same. The resonance of the fabricated structure at $0.5 \mathrm{THz}$ was verified and mathematically conformed.Further the fabricated structure can be tested using Terahertz-TDS spectroscopy for its resonant operation and the radiation pattern characteristics can be studied using the same by scanning the sample at different orientations and angles.

Table 4 Radiation Characteristics of the Laser patterned antenna

\begin{tabular}{|c|c|c|c|c|}
\hline Type & $\begin{array}{l}\text { Frequency } \\
\text { (THz) }\end{array}$ & $\begin{array}{l}\text { S11 } \\
\text { (dB) }\end{array}$ & $\begin{array}{l}\text { Gain } \\
\text { (dB) }\end{array}$ & $\begin{array}{l}D \\
\text { (dB) }\end{array}$ \\
\hline Patch & $33^{0.15,0.2}$ & $\begin{array}{l}-31.46, \\
-27.22\end{array}$ & 5.17 & 5.3 \\
\hline
\end{tabular}

\section{THz-TDS Measurement}

A Conventional measurement system using Vector Network Analyser (VNA) is restricted to low frequencies due to the antenna size factor. At higher frequencies, THz-TDS measurement is used to determine the resonance of the antenna structure.

In this, THz-TDS technique is adopted for the fabricated copper based antenna to confirm its resonance. The terahertz pulse used for the measurement is shown in Fig.17.This setup uses BATOP (Photoconductive Antenna) to generate $\mathrm{THz}$ radiation from the Femtosecond Laser source (10fs, $800 \mathrm{~nm}$ wavelength, $78 \mathrm{MHz}$ repetition rate) and a beam splitter is used to generate pump and probe signal. The setup uses ZnTe crystal for $\mathrm{THz}$ detection.Fig.18. Shows the transmission spectra of the copper-based structure measured using THz-TDS system. The measurement shows that it resonates at $0.12 \mathrm{THz}$. The deviation in the resonance is because of thermal diffusion of the ablated samples by the laser. Thus the resonance of the fabricated structure is validated experimentally.

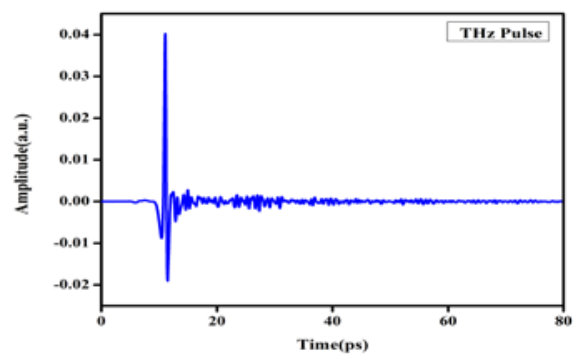

Fig.17 THz Pulse 


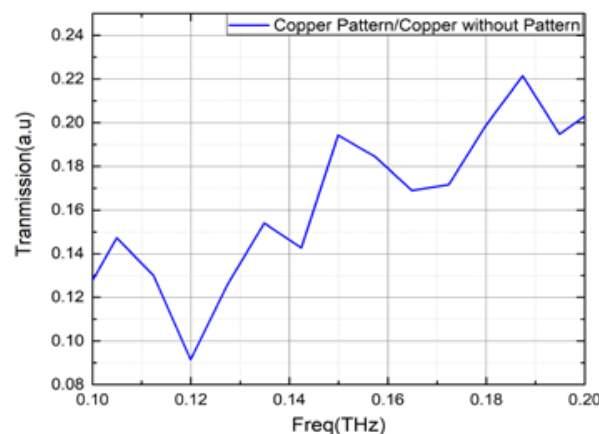

Fig.18 THz-TDS Transmission spectra for the copper based structure

\section{Conclusions}

The experimental details of nanosecond and picoseconds laser pulses ablation of Copper and ITO thin films have been studied and discussed. The observation shows the creation of patch structure on ITO at a particular Fluence of $0.87 \mathrm{~J} / \mathrm{cm}^{2}$ with optimized scanning speed, while copper requires the Fluence of $4.33 \mathrm{~J} / \mathrm{cm}^{2}$ in ns laser ablation. A comparatively small quantum of energy needs to be supplied for ablation of the same depth in comparison with ns pulsed laser, for picoseconds pulses. The morphology and ablation rate characteristics were analyzed using SEM, Optical Profiler and White Line Interferometer.FEM numerical modeling has been done for ns pulsed laser ablation of $\mathrm{Cu}$, ITO and its temperature distributions, stress \& strain have been discussed. Finally, an application of the created pattern as Terahertz Antenna has been analyzed and validated experimentally.

\section{References}

[1] E.Manikandan, G.Padmalaya, B.S.Sreeja and S.Radha: Water and Energy International., 59, (2017) 39.

[2] T. W. Turpin and R. Baktur: IEEE Antennas Wireless Propag. Lett., 8,(2009) 693.

[3] S.Ananad, D.Sriram kumar: Physica E Low Dimens Syst Nanostruct., 66, (2015) 52.

[4] J. Hautcoeur, F. Colombel, X. Castel, M. Himdi, and E. M. Cruz: PIER C., 22, (2011) 259.

[5] D.Mittleman, M.Gupta and R.Neelamani: Appl Phys B., 68, (1999) 1085.

[6] J. D. Mahony: IEEE Antennas Propag. Mag., 33, (1991) 51.

[7] DA. Willis and X.Xu: Int. J. Heat Mass Transfer., 45, (2002) 3911.

[8] A.Bogaerts and Z.Chen: Spectrochim. Acta, Part B., 60, (2005) 1280.

[9] B.S. Shin, J.Y. Oh and H. Sohn: J. Mater. Process. Technol., 187, (2007) 260.

[10] S.Y. Wang, Y.P. Ren, K.P. Chang, C.W. Cheng, J.K. Chen, and D.Y. Tzou: J Laser Micro/Nanoengin., 9,(2014) 88.

[11] A.Bogaerts, Z.Chen and D.Bleiner: J. Anal. At. Spectrom., 21, (2006) 384.
[12]K. K. Anoop, R. Fittipaldi, A. Rubano, X. Wang, D. Paparo, A. Vecchione, L. Marrucci, R. Bruzzese, and S. Amoruso: J. Appl. Phys., 116, (2014) 113102.

[13] M. Aghaei, S. Mehrabian, and S. H. Tavassoli: J. Appl. Phys., 104, (2008), 053303.

[14] J.Mur, B.Podobnik and I.Poberaj: Opt Laser Technol., 88, (2017) 140.

[15] O. Yavas and M. Takai: Appl. Phys. Lett., 73, (1998) 2558

[16] A.Risch and R.Hellmann: Phys. Procedia., 12, (2011) 133.

[17] SJ.

Henley, M.Cann, I.Jurewicz, A.Dalton and D.M ilne: Nanoscale., 6, (2014) 946.

[18] S.Aart, S.Viktor, H.Oliver, and S.Uwe: J Laser Micro/Nanoengin., 5,(2010),248.

[19] C. McDonnell, D.Milne, C. Prieto, H. Chan, D. Rostohar and G.M. O’Connor: Appl. Surf. Sci., 359, (2015) 567.

[20] X. C. Wang, H. Y. Zheng, C. W. Tan, F. Wang, H. Y. Yu, and K. L. Pey: Opt. Express.,18, (2010) 19379.

[21]K.S.Tseng and Y.L. Lo: Opt. Mater. Express., 4, (2014) 764.

(Received: August 12, 2017, Accepted: Ocrober 17, 2017) 